\title{
Lessons learned from extracorporeal membrane oxygenation as a bridge to lung transplantation
}

\author{
Vladimir Shumaster, ${ }^{1,2}$ Oliver K. Jawitz, ${ }^{1}$ David Yuh, ${ }^{2}$ and Pramod Bonde*,1,2,3 \\ ${ }^{1}$ Bonde Artificial Heart Laboratory, Yale University School of Medicine, New Haven, CT, USA \\ ${ }^{2}$ Section of Cardiac Surgery, Yale-New Haven Hospital, Yale University School of Medicine, New Haven, CT, USA \\ ${ }^{3}$ Yale Center for Advanced Heart Failure, Mechanical Support, and Heart Transplantation, Yale University School of \\ Medicine, New Haven, CT, USA \\ *Corresponding author's e-mail address: pramod.bonde@yale.edu
}

Published online: May 16, 2014 (version 1)

Cite as: Shumaster et al., ScienceOpen Research 2014 (DOI: 10.14293/A2199-1006.01.SOR-MED.BG1R6.v1)

Reviewing status: Please note that this article is under continuous review. For the current reviewing status and the latest referee's comments please click here or scan the QR code at the end of this article.

Primary discipline: Medicine

Keywords: Extracorporeal membrane oxygenation, ECMO, Lung transplantation, Bridge to lung transplantation

\begin{abstract}
Extracorporeal membrane oxygenation (ECMO) has been used infrequently as a bridge to lung transplantation due to lack of consensus and data regarding the benefits of such a strategy. We present data from the United Network of Organ Sharing (UNOS) database on the outcomes of patients bridged to lung transplantation with ECMO. We used the UNOS database to analyze data between January 1, 2000 and December 31,2011 . During this time 14,263 lung transplants were performed, of which 143 (1.0\%) were bridged using ECMO. Patients on ECMO as a bridge to lung transplantation were compared to those transplanted without prior ECMO support. Demographics, survival rates, complications, and rejection episodes were compared between the two groups. The 30day, 6-month, 1-year, 3-year, and 5-year survival rates were $69 \%, 56 \%, 48 \%, 26 \%$, and $11 \%$, respectively, for the ECMO bridge group and 95\%, 88\%, 81\%, 58\%, and 38\% respectively, for the control group $(p \leq 0.01)$. The ECMO group incurred higher rate of postoperative complications, including airway dehiscence ( $4 \%$ vs. $1 \%, p \leq 0.01$ ), stroke ( $3 \%$ vs. $2 \%$, $p \leq 0.01$ ), infection ( $56 \%$ vs. $42 \%, p \leq 0.01$ ), and pulmonary embolism ( $10 \%$ vs. $0.6 \%, p \leq 0.01$ ). The length of hospital stay was longer for the ECMO group (41 vs. 25 days, $p \leq$ $0.01)$, and they were treated for rejection more often $(49 \%$ vs. $36 \%, p=0.02$ ). The use of ECMO as a bridge to lung transplantation is associated with significantly worse survival and more frequent postoperative complications. Therefore, we advocate very careful patient selection and cautious use of ECMO.
\end{abstract}

Presented at the New England Surgical Society, 94th Annual Meeting, Hartford, CT, September 2013.

\section{INTRODUCTION}

With the demand for donor lungs continuously outpacing their availability, transplant surgeons have long sought temporizing measures to sustain recipients awaiting transplantation. Although mechanical ventilation has been used as a conventional bridging modality, certain pathologic aspects of candidates awaiting lung transplantation limit the practical utility of this option (e.g., idiopathic pulmonary fibrosis and primary pulmonary hypertension) including chronic airway damage resulting from barotrauma associated with prolonged positive pressure ventilation. Heretofore, extracorporeal membrane oxygenation (ECMO) has been used infrequently as an alternative bridge to lung transplantation with mixed outcomes [1-2]. Within the past few years, however, there has been renewed interest in using this bridging technology in waiting recipients who otherwise would not have survived. Advantages of ECMO over mechanical ventilation as a bridging modality lie in its capacity to not only rest the lung parenchyma and avoid the complications associated with prolonged intubation but also to reliably achieve full oxygenation and adequate carbon dioxide removal.

Prior single institution studies have been encouraging but handicapped with small patient cohorts [1-6]. We present data from the United Network of Organ Sharing (UNOS) database on the outcomes of patient bridged to lung transplantation using ECMO to reflect the global experience among centers applying this bridging strategy. This data can be used to help refine application of ECMO to this challenging patient population.

\section{MATERIAL AND METHODS}

The Yale University School of Medicine Institutional Review Board approved this study. A retrospective analysis of the UNOS database, from January 2000 to December 2011, was performed to compare the outcomes of lung and heart-lung 
Table 1. Donor characteristics.

\begin{tabular}{|c|c|c|c|c|c|}
\hline & \multicolumn{2}{|c|}{ ECMO group } & \multicolumn{2}{|c|}{ No ECMO group } & \multirow[b]{2}{*}{$p$-value } \\
\hline & Average $(n=143) \pm$ SD & Range & Average $(n=14120) \pm$ SD & Range & \\
\hline Age (years) & $34.3 \pm 14.3$ & $10-73$ & $33.1 \pm 14.1$ & $2-76$ & 0.324 \\
\hline \multicolumn{6}{|l|}{ Gender } \\
\hline Male & $73(51.05 \%)$ & & $8471(60.0 \%)$ & & \\
\hline Female & 70 (48.95\%) & & $5649(40.0 \%)$ & & 0.0299 \\
\hline BMI & $25.5 \pm 5.3$ & $18.1-43.6$ & $25.1 \pm 4.9(14107)$ & $10.8-54.9$ & 0.2754 \\
\hline pO2 & $397.5 \pm 153.4$ & $47.0-619$ & $402.2 \pm 142.2$ & $30.7-754.0$ & 0.693 \\
\hline \multicolumn{6}{|l|}{ Donor type } \\
\hline Deceased & $143(100 \%)$ & & $14060(99.6 \%)$ & & 0.4347 \\
\hline History of cigarette use & $25(17.48 \%)$ & & $2486(17.67 \%)$ & & 0.998 \\
\hline Donor pulmonary infection & $52(36.4 \%)$ & & $4249(30.2 \%)$ & & 0.1117 \\
\hline Donor inotropic medications at procurement & $81(57.5 \%)$ & & $8533(63.1 \%)$ & & 0.3706 \\
\hline Donor cause of death & $n=143$ & & $n=14058$ & & 0.0668 \\
\hline Anoxia & $24(16.78 \%)$ & & 1347 (9.58\%) & & \\
\hline Cerebrovascular/stroke & $47(32.87 \%)$ & & $5061(36.0 \%)$ & & \\
\hline Head trauma & 67 (46.85\%) & & $7208(51.27 \%)$ & & \\
\hline CNS tumor & $1(0.7 \%)$ & & $126(0.9 \%)$ & & \\
\hline Other & $4(2.8 \%)$ & & $316(2.25 \%)$ & & \\
\hline Donor mechanism of death & $n=143$ & & $n=14086$ & & 0.0179 \\
\hline Drowning & $0(0 \%)$ & & $29(0.2 \%)$ & & \\
\hline Seizure & $2(1.4 \%)$ & & $123(0.9 \%)$ & & \\
\hline Drug intoxication & 7 (4.9\%) & & $353(2.5 \%)$ & & \\
\hline Asphyxiation & $5(3.5 \%)$ & & $276(2.0 \%)$ & & \\
\hline Cardiovascular & $13(9.1 \%)$ & & $651(4.4 \%)$ & & \\
\hline Gunshot wound & $17(11.9 \%)$ & & $2869(20.4 \%)$ & & \\
\hline Stab & $0(0 \%)$ & & $21(0.2 \%)$ & & \\
\hline Blunt injury & $45(31.5 \%)$ & & $4004(28.5 \%)$ & & \\
\hline Intracranial hemorrhage/stroke & $48(33.6 \%)$ & & 5306 (37.7\%) & & \\
\hline Death from natural causes & $0(0 \%)$ & & $146(1.0 \%)$ & & \\
\hline None of the above & $6(4.2 \%)$ & & $308(2.2 \%)$ & & \\
\hline
\end{tabular}

transplant recipients that were bridged to transplant with ECMO versus those recipients that were not (control group). During these 11 years, 14,263 lung transplants were performed, of which, 143 patients $(1.0 \%)$ were bridged to transplant using ECMO. Both primary and retransplants were included. Only patients 18 years old or older were included in the study. As the database does not separate veno-arterial from veno-venous ECMO, all bridged patients were included regardless of the type of ECMO. Donor and recipient characteristics were compared for each group with univariate and multivariate analysis using SAS 9.3 (Cary, NC, USA) statistical software.

The primary end points included 30-day, 3-month, 6-month, 1-year, 2-year, 3-year, 5-year, and 10-year mortalities. Continuous variables are presented as a mean \pm SD and the range. Categorical variables are displayed as frequency of distribution ( $n$ ) and percentage (\%). Kaplan-Meier curves were used to show survival outcomes. The $p$-values for continuous variables were calculated using a Student's $t$-test, and chisquare analysis was used for categorical variables. For all comparisons, two-tailed $p$-values less than 0.05 were considered statistically significant. Multivariate analysis was performed using the Cox proportional hazards model. All variables that showed statistically significant results using univariate analysis were included in the multivariate analysis.

\section{RESULTS \\ Donor characteristics}

Donor characteristics were similar between the two groups (Table 1) with the exception that there was a higher percentage of lungs from male donors that were transplanted into the control group $(60.0 \%$ vs. $51.1 \%, p=0.03)$. Multivariate analysis did not show this to be statistically significant ( $p=$ 0.389, HR: 0.96 , 95\% CI: 0.875-1.05). The causes of death among donors was not statistically significant between the two groups ( $p=0.07$ ) with the most common causes for both groups being head injury and stroke accounting for $79.72 \%$ of lungs used in the ECMO group, and $87.3 \%$ in the control group (Table 1). There were a higher percentage of lungs used from patients that died from anoxia in the ECMO group (16.8\% vs. 9.6\%). The causative mechanisms of death among the donors were also similar between the two groups, with the most common mechanisms being gunshot wounds, blunt injury, and stroke. Notably, $3.5 \%$ of donor lungs in the ECMO group and $2.0 \%$ of donor lungs in the control group were taken from people who died of asphyxiation. 
Table 2. Recipient characteristics.

\begin{tabular}{|c|c|c|c|c|c|}
\hline & \multicolumn{2}{|l|}{ ECMO group } & \multicolumn{2}{|l|}{ No ECMO group } & \multirow[b]{2}{*}{$p$-value } \\
\hline & Average $(n=143) \pm$ SD & Range & Average $(n=14120) \pm$ SD & Range & \\
\hline Age (years) & $47.3 \pm 15.0$ & $18-74$ & $52.2 \pm 13.0$ & $18-81$ & $<0.0001$ \\
\hline \multicolumn{6}{|l|}{ Gender } \\
\hline Male & $80(55.9 \%)$ & & $7783(55.1 \%)$ & & \multirow[b]{2}{*}{0.8438} \\
\hline Female & $63(44.1 \%)$ & & $6337(44.9 \%)$ & & \\
\hline BMI Recipient & $25.0 \pm 5.3$ & $13.7-37.2$ & $24.5 \pm 4.8$ & $7.3-54.0$ & 0.2154 \\
\hline Diabetes & $29(20.3 \%)$ & & 2107 (14.9\%) & & 0.074 \\
\hline History of cigarette use & $51(46.4 \%)$ & & $5331(62.0 \%)$ & & 0.0008 \\
\hline Chronic steroid use & $76(53.2 \%)$ & & $6688(47.4 \%)$ & & 0.0575 \\
\hline Pretransplant dialysis since listing & $17(11.9 \%)$ & & $56(0.4 \%)$ & & $<0.0001$ \\
\hline History of malignancy & $10(7.0 \%)$ & & $682(4.8 \%)$ & & $<0.0001$ \\
\hline Lung surgery Between listing and transplant (nontransplant) & $30(21.0 \%)$ & & $1105(7.8 \%)$ & & $<0.0001$ \\
\hline Prior lung surgery & $8(15.7 \%)$ & & $1318(16.6 \%)$ & & 0.4313 \\
\hline Previous transplants & $27(18.9 \%)$ & & $519(3.7 \%)$ & & $<0.0001$ \\
\hline Days between previous and current transplant & $516.1 \pm 1166.2$ & $1.0-4729$ & $1518.5 \pm 1299.5$ & $1.0-6898$ & $<0.0001$ \\
\hline Days waiting from initial date to end date & $170.4 \pm 336.9$ & $0-2151$ & $319.7 \pm 447.7$ & $0-5843$ & $<0.0001$ \\
\hline PA mean pressures (mmHg) & $32.1 \pm 14.7$ & $4.0-84.0$ & $27.6 \pm 11.8$ & $0.0-110.0$ & 0.0002 \\
\hline Pulmonary capillary wedge pressures $(\mathrm{mmHg})$ & $11.7 \pm 7.9$ & $1.0-39.0$ & $11.2 \pm 5.9$ & $0.0-50.0$ & 0.4143 \\
\hline Cardiac output (L/min) & $5.3 \pm 1.8$ & $2.0-15.0$ & $5.3 \pm 1.5$ & $0.2-15.0$ & 0.8576 \\
\hline $\mathrm{O}_{2}$ requirements $(\mathrm{L} / \mathrm{min})$ & $8.0 \pm 7.0$ & $0-20$ & $2.9 \pm 2.4$ & $0-20$ & $<0.0001$ \\
\hline $\mathrm{pCO}_{2}(\mathrm{mmHg})$ & $47.2 \pm 16.8$ & 10.0-107.0 & $46.6 \pm 12.7$ & $10.0-120.0$ & 0.7105 \\
\hline FEV1 (\%) & $42.5 \pm 22.0$ & 11.0-105.0 & $36.5 \pm 21.0$ & $5.0-120.0$ & 0.0059 \\
\hline FVC (\%) & $47.8 \pm 19.8$ & 11.0-98.0 & $49.7 \pm 17.7$ & 10.0-130.0 & 0.3079 \\
\hline Creatinine & $1.08 \pm 0.81$ & $0.20-7.01$ & $0.90 \pm 0.52$ & $0.10-20.0$ & $<0.0001$ \\
\hline Total bilirubin (mg/dL) & $1.25 \pm 2.09$ & $0.1-21.4$ & $0.72 \pm 1.59$ & $0.1-82.0$ & 0.0001 \\
\hline Episodes of ventilator support & $76(53.2 \%)$ & & $801(5.7 \%)$ & & $<0.0001$ \\
\hline Life support & $143(100 \%)$ & & $973(6.9 \%)$ & & $<0.0001$ \\
\hline Ischemia time (Hours) & $5.43 \pm 1.87$ & $0.2-11.75$ & $4.90 \pm 1.70$ & $0.17-12.62$ & 0.0003 \\
\hline Length of hospital stay (transplant to discharge (days) & $41.2 \pm 45.4$ & $0-261$ & $24.5 \pm 30.3$ & $0-566$ & $<0.0001$ \\
\hline Lung allocation score at match & $68.9 \pm 22.9$ & $28.4-95.2$ & $44.2 \pm 15.1$ & $0-95.5$ & $<0.0001$ \\
\hline Transplant type & $n=129$ & & $n=13820$ & & 0.0004 \\
\hline Single lung & $34(26.4 \%)$ & & $5764(41.7 \%)$ & & \\
\hline Double lung & $95(73.6 \%)$ & & $8056(58.3 \%)$ & & \\
\hline
\end{tabular}

\section{Recipient characteristics}

The recipients in the ECMO group tended to be younger, with an average age of 47 years versus 52 years in the control group ( $p \leq 0.01$ ) (Table 2). The control group recipients were more likely to have a history of tobacco use $(62 \%$ versus $47 \%, p=0.01$ ). This, however, was not shown to be significant using multivariable analysis. ECMO recipients also were more likely to require pretransplant dialysis $12 \%$ versus $0.4 \%$ ( $p \leq 0.01$ ), more likely to have a history of malignancy $7 \%$ versus $5 \%$ ( $p \leq 0.01$ ), more likely to have had prior lung surgery (nontransplant) between the time they were listed and the time they were transplanted $(21 \%$ versus $8 \%, p \leq$ 0.01 , and more likely to have had a previous lung transplant (19\% versus $4 \%, p \leq 0.01)$. None of these differences, however, were found to be statistically significant by multivariate analysis (Table 3).

ECMO patients were generally sicker with higher mean pulmonary arterial pressures and supplemental oxygen requirements. They were also more likely to be on some type of life support (i.e., mechanical ventilation, inotropes), and their hospital stays were longer (41 days versus 25 days, $p \leq$ 0.01). Fifty-one percent of ECMO patients were mechanically ventilated prior to transplant versus just $4 \%$ in the control group ( $p \leq 0.01$ ). As would be expected, the Lung Allocation Score (LAS) was higher for the ECMO group ( $p \leq 0.01)$. ECMO patients were also more likely to receive a double-lung transplant as opposed to a single-lung transplant $(74 \%$ versus $58 \%, p=0.01)$, and had greater graft ischemic times ( $p=$ 0.01). The most common diagnoses among transplant recipients were Idiopathic Pulmonary Fibrosis (IPF), Chronic Obstructive Lung Disease (COPD)/Emphysema, and Cystic Fibrosis (Table 4). The most notable difference in the preoperative diagnoses was that $9.1 \%$ of ECMO patients and only $0.5 \%$ of control group patients were retransplanted for acute rejection/primary graft failure.

\section{Survival}

The 30-day, 90-day, 6-month, 1-year, 3-year, 5-year, and 10year survival rates were $68.5 \%, 59.4 \%, 55.9 \%, 47.7 \%, 25.5 \%$, 
Table 3. Multivariate analysis.

\begin{tabular}{|c|c|c|c|c|}
\hline & Hazards ratio & 95\% confidence interval & Standard error & $p$-value \\
\hline Donor gender & 1.072 & $0.968-1.187$ & 0.05 & 0.1808 \\
\hline Recipient age & 1.012 & $1.006-1.018$ & 0.003 & $<0.0001$ \\
\hline History of cigarette use & 0.934 & $0.83-1.051$ & 0.06 & 0.2591 \\
\hline Pretransplant dialysis—since listing & 1.34 & $0.724-2.478$ & 0.31 & 0.3513 \\
\hline Previous malignancy & 1.029 & $0.86-1.231$ & 0.09 & 0.756 \\
\hline Previous transplant & 0.986 & $0.677-1.437$ & 0.19 & 0.9411 \\
\hline Transplant type (single lung) & 0.957 & $0.853-1.075$ & 0.06 & 0.4628 \\
\hline Ischemia time & 1.015 & $0.985-1.045$ & 0.02 & 0.3404 \\
\hline Lung allocation score at match & 1.009 & $1.005-1.013$ & 0.002 & $<0.0001$ \\
\hline Lung surgery between listing and transplant (nontransplant) & 0.956 & $0.814-1.123$ & 0.08 & 0.5855 \\
\hline Life support & 0.469 & $0.221-0.993$ & 0.38 & 0.048 \\
\hline Intraortic balloon pump & 5.368 & $0.583-49.421$ & 1.13 & 0.1379 \\
\hline Prostacyclin infusion & 2.218 & $0.832-5.441$ & 0.48 & 0.115 \\
\hline Prostacyclin inhalation & 4.067 & $1.30-12.725$ & 0.58 & 0.0159 \\
\hline Inhaled nitric oxide & 0.709 & $0.319-1.578$ & 0.41 & 0.3994 \\
\hline Ventilator support & 2.698 & $1.262-5.767$ & 0.39 & 0.0104 \\
\hline Other life support & 2.81 & $1.357-5.818$ & 0.37 & 0.0054 \\
\hline Episodes of ventilator support & 0.903 & $0.724-1.125$ & 0.11 & 0.3625 \\
\hline PA mean pressures & 1.005 & $1.000-1.010$ & 0.003 & 0.356 \\
\hline FEV1 & 1.003 & $1.000-1.006$ & 0.002 & 0.0838 \\
\hline Creatinine & 1.051 & $0.98-1.128$ & 0.04 & 0.1632 \\
\hline Total bilirubin & 1.048 & $1.026-1.071$ & 0.01 & $<0.0001$ \\
\hline Length of hospital stay (Transplant to discharge [days]) & 1.002 & $1.001-1.003$ & 0 & 0.0002 \\
\hline
\end{tabular}

$11.2 \%$, and $0 \%$, respectively, for the ECMO group and $95.1 \%$, $91.3 \%, 87.6 \%, 81.0 \%, 57.5 \%, 38.4 \%$, and $5.1 \%$, respectively, for the control group ( $p \leq 0.01$ ) (Table 5). Kaplan-Meier curves for both groups are illustrated in Figure 1. Although

Table 4. Recipient diagnosis.

\begin{tabular}{|c|c|c|c|}
\hline & $\begin{array}{c}\text { ECMO } \\
\text { group } \\
(n=122)\end{array}$ & $\begin{array}{l}\text { No ECMO } \\
\text { group } \\
(n=13,194)\end{array}$ & $p$-value \\
\hline \multicolumn{4}{|l|}{ Diagnosis (most common) } \\
\hline Scleroderma-restrictive & $2(1.4 \%)$ & $41(0.3 \%)$ & $<0.0001$ \\
\hline $\begin{array}{l}\text { Scleroderma_pulmonary } \\
\text { hypertension }\end{array}$ & $3(2.1 \%)$ & $78(0.6 \%)$ & \\
\hline Hypersensitivity pneumonitis & $2(1.4 \%)$ & $55(0.4 \%)$ & \\
\hline Lupus & $2(1.4 \%)$ & $5(0.04 \%)$ & \\
\hline $\begin{array}{l}\text { Lung retransplant/graft failure: } \\
\text { obliterative bronchiolitis }\end{array}$ & $7(4.9 \%)$ & $271(1.9 \%)$ & \\
\hline $\begin{array}{l}\text { Lung retransplant/graft failure: } \\
\text { acute rejection }\end{array}$ & $2(1.4 \%)$ & $10(0.07 \%)$ & \\
\hline $\begin{array}{l}\text { Lung retransplant/graft failure: } \\
\text { primary graft failure }\end{array}$ & $13(9.1 \%)$ & $71(0.5 \%)$ & \\
\hline $\begin{array}{l}\text { Primary pulmonary } \\
\text { hypertension }\end{array}$ & $6(4.2 \%)$ & $394(2.8 \%)$ & \\
\hline Cystic fibrosis & $12(8.4 \%)$ & $1811(12.8 \%)$ & \\
\hline $\begin{array}{l}\text { Idiopathic pulmonary } \\
\text { Fibrosis/usual interstitial } \\
\text { Pneumonitis }\end{array}$ & $38(26.6 \%)$ & $3786(26.8 \%)$ & \\
\hline Sarcoidosis & $3(2.1 \%)$ & $454(3.2 \%)$ & \\
\hline Alpha-1 antitrypsin deficiency & $2(1.4 \%)$ & $599(4.2 \%)$ & \\
\hline COPD/emphysema & $21(14.7 \%)$ & $4564(32.3 \%)$ & \\
\hline Bronchiectasis & $1(0.7 \%)$ & $251(1.8 \%)$ & \\
\hline Pulmonary fibrosis - other & $6(4.2 \%)$ & $466(3.3 \%)$ & \\
\hline Other & $2(1.4 \%)$ & $338(2.4 \%)$ & \\
\hline
\end{tabular}

the donor and the recipient characteristics were similar, patients bridged with ECMO were, in general, sicker requiring ventilator support while on the list $53.2 \%$ of the time versus $5.7 \%$ in the control group $(p \leq 0.01)$ and more frequently required other forms of life support.

When survival outcomes were separated out on the basis of prior transplant, retransplant patients exhibited worse survival in both groups (Figure 2). Similarly, survival was worse for the patients receiving a single-lung transplant compared to patients receiving a double-lung transplant in either of the groups (Figure 3).

\section{Postoperative complications}

The ECMO group experienced a significantly higher rate of postoperative complications, including the need for blood

Table 5. Survival.

\begin{tabular}{lccc}
\hline & $\begin{array}{c}\text { ECMO group } \\
(\boldsymbol{n}=\mathbf{1 4 3})\end{array}$ & $\begin{array}{c}\text { No ECMO group } \\
(\boldsymbol{n}=\mathbf{1 4 1 1 7})\end{array}$ & $\boldsymbol{p}$-value \\
\hline Patient survival & & & \\
30 days & $98(68.53 \%)$ & $13,419(95.1 \%)$ & $<0.0001$ \\
90 days & $85(59.4 \%)$ & $12,883(91.3 \%)$ & $<0.0001$ \\
6 months & $80(55.9 \%)$ & $12,339(87.6 \%)$ & $<0.0001$ \\
1 year & $62(47.7 \%)$ & $10,938(81.0 \%)$ & $<0.0001$ \\
2 years & $36(31.6 \%)$ & $8329(68.3 \%)$ & $<0.0001$ \\
3 years & $27(25.47 \%)$ & $6409(57.5 \%)$ & $<0.0001$ \\
5 years & $11(11.2 \%)$ & $3687(38.4 \%)$ & $<0.0001$ \\
10 years & $0(0 \%)$ & $375(5.1 \%)$ & 0.027 \\
\hline
\end{tabular}




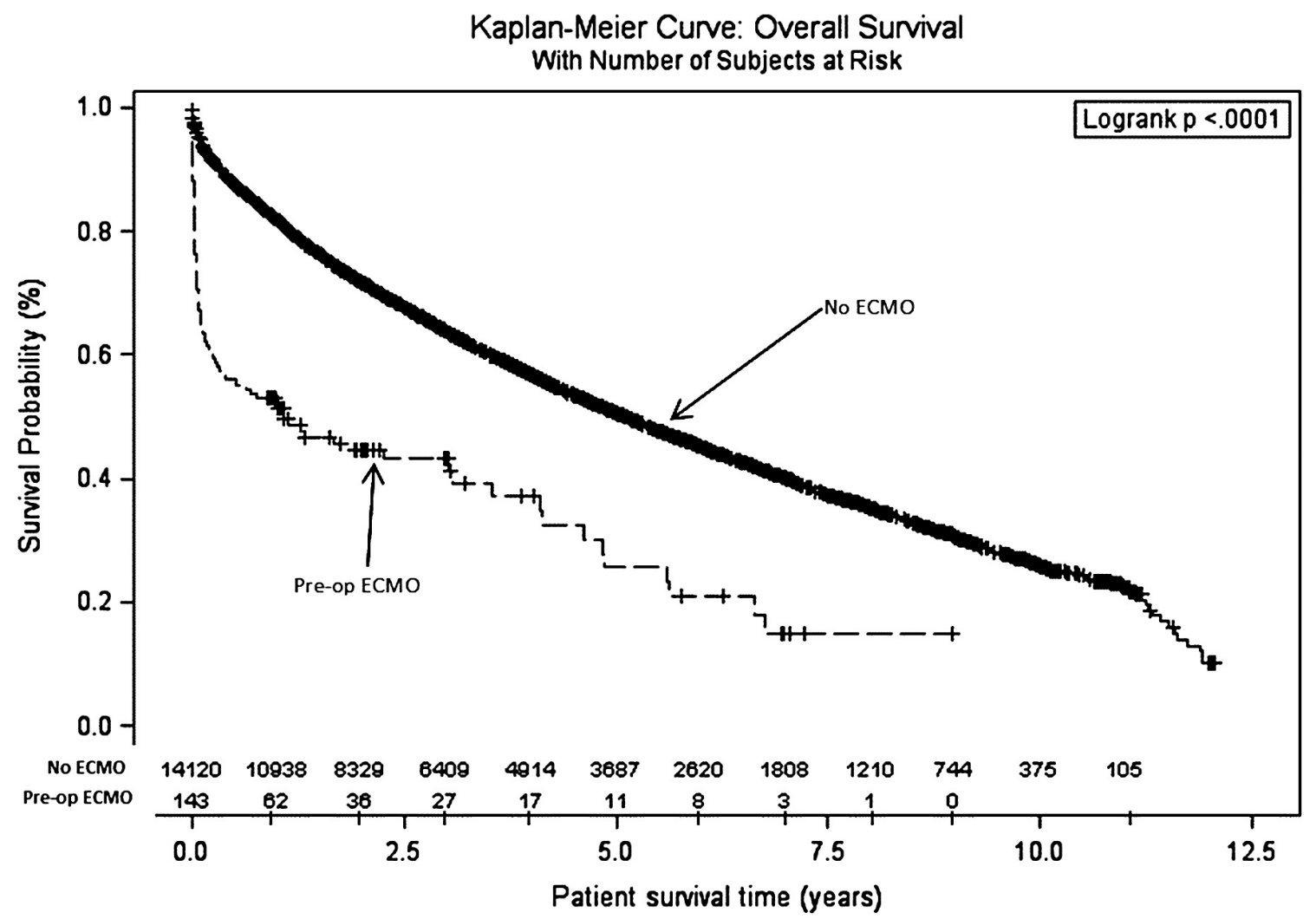

Figure 1. Kaplan-Meier Curve: Comparing survivals of patients on pretransplant ECMO with those that were not on ECMO.

transfusions $(34.3 \%$ versus $4.4 \%, p \leq 0.01)$, airway dehiscence $(4.2 \%$ versus $1.3 \%, p \leq 0.01)$, stroke $(2.8 \%$ versus $1.9 \%, p \leq 0.01)$, need for dialysis $(31.5 \%$ versus $5.8 \%$, $p \leq 0.01)$, infections requiring antibiotics $(55.9 \%$ versus
$42.4 \%, p \leq 0.01$ ), pulmonary embolisms $9.8 \%$ versus $0.6 \%$, $p \leq 0.01$ ), and the need for other posttransplant surgical procedures $(60.8 \%$ versus $19.1 \%, p \leq 0.01$ ) (Table 6). ECMO patients tended to have a slightly higher rate of acute

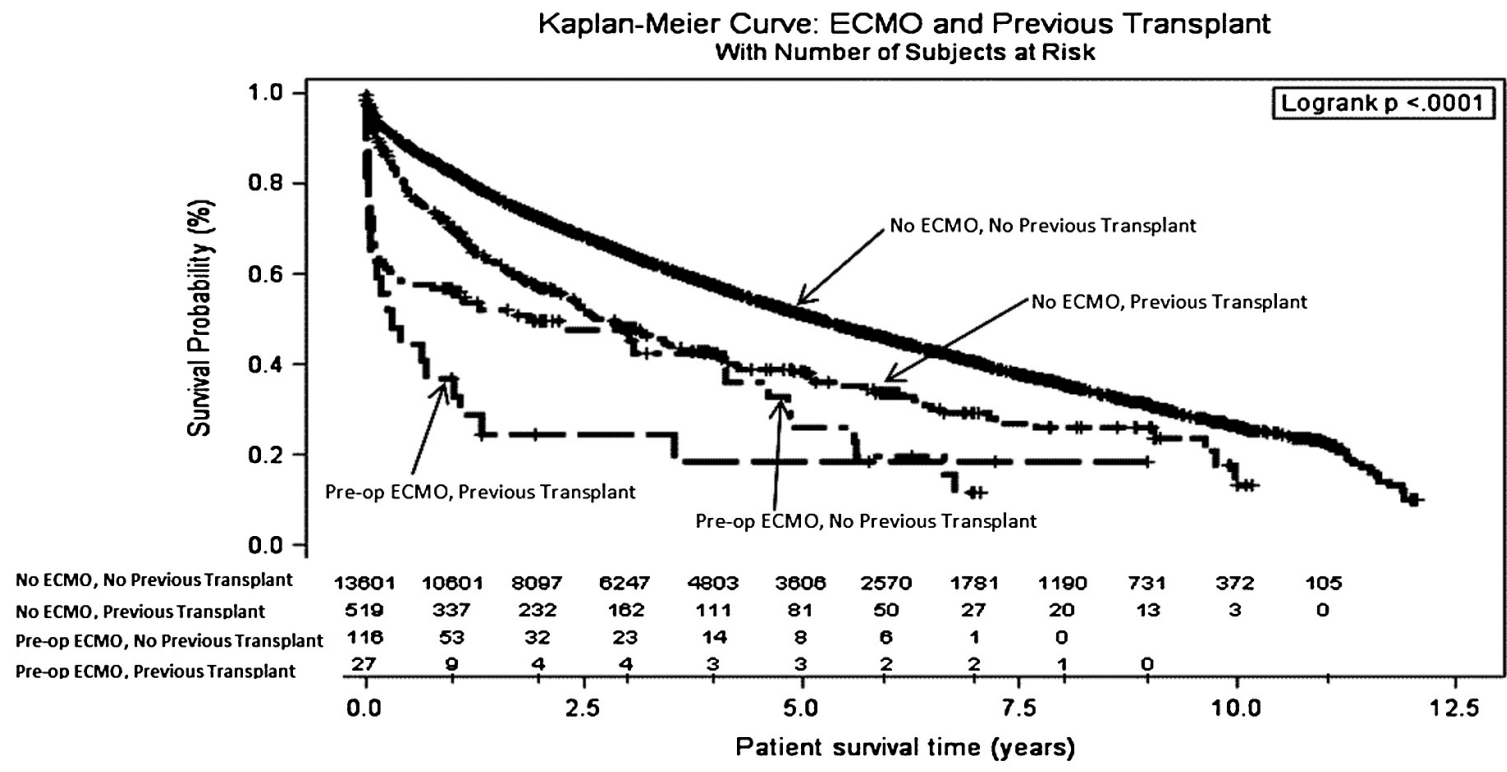

Figure 2. Kaplan-Meier Curve: Comparing survivals of patients on pretransplant ECMO with those that were not on ECMO who either had or did not have a previous transplant. 


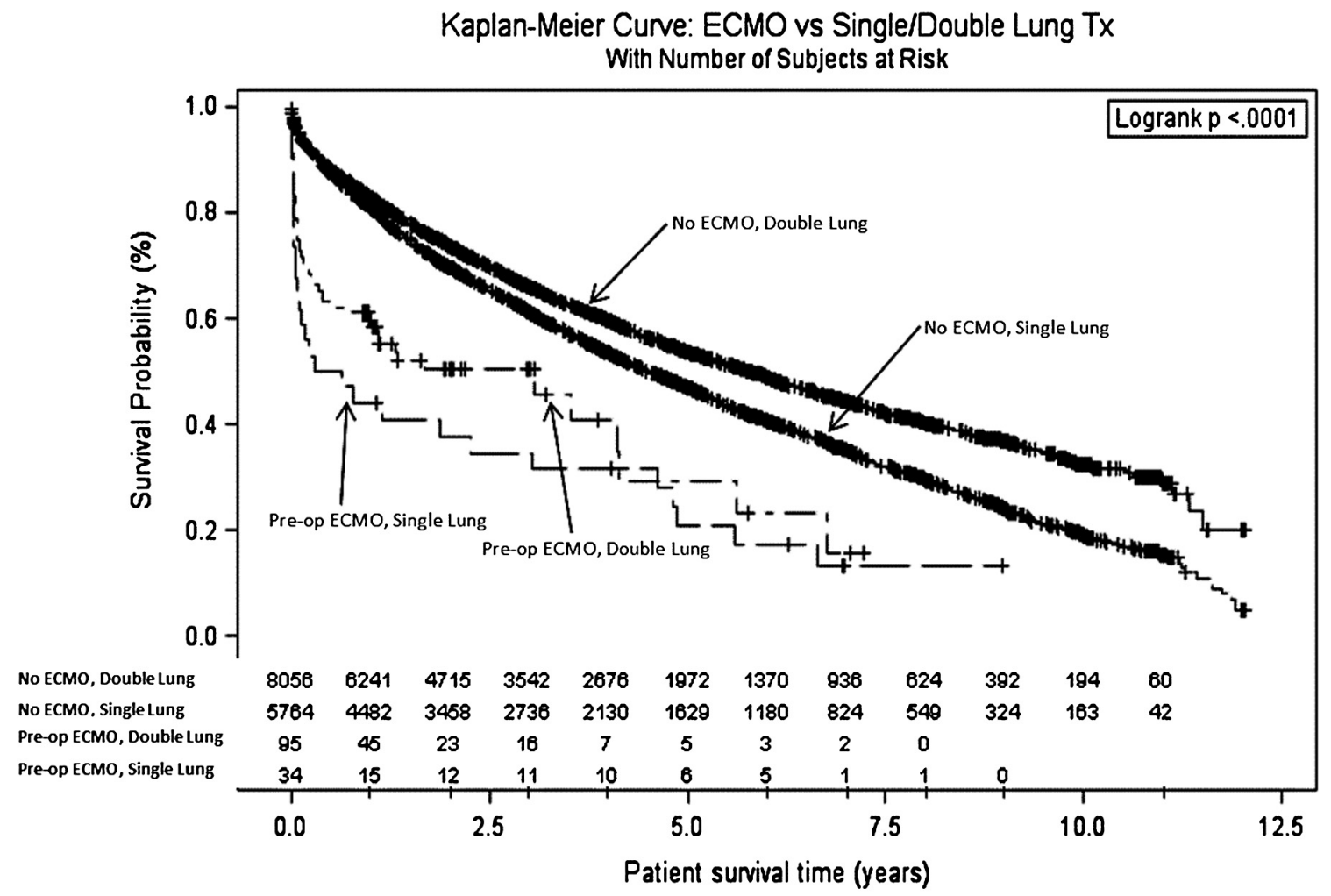

Figure 3. Kaplan-Meier Curve: Comparing survivals of patients on pretransplant ECMO with those that were not on ECMO separated by type of transplant performed (single vs double lung).

rejection episodes posttransplant $10.5 \%$ versus $6.9 \%$, though this was not found to be statistically significant $(p=0.09)$ (Tables 7-8).

\section{DISCUSSION}

In this study, we analyzed the UNOS database to compare the outcomes of patients who were bridged to lung transplantation with ECMO to recipients who were not over an 11-year span. We believe this to be the largest multicenter cohort of ECMO-bridged lung transplant recipients to date. Our analysis suggests that there is no survival benefit afforded to lung transplant recipients bridged with ECMO. Contrary to many

Table 6. Complications.

\begin{tabular}{lccc}
\hline & $\begin{array}{c}\text { ECMO group } \\
(\boldsymbol{n}=\mathbf{1 4 3 )}\end{array}$ & $\begin{array}{c}\text { No ECMO group } \\
(\boldsymbol{n}=\mathbf{1 4 , 1 2 0 )})\end{array}$ & $\boldsymbol{p}$-value \\
\hline Airway dehiscence & $6(4.2 \%)$ & $186(1.3 \%)$ & $<0.0001$ \\
$\begin{array}{l}\text { Stroke (posttransplant) } \\
\text { Dialysis (posttransplant) }\end{array}$ & $45(2.8 \%)$ & $265(1.9 \%)$ & $<0.0001$ \\
$\begin{array}{l}\text { Any drug treated infec- } \\
\quad \text { tion (posttransplant) }\end{array}$ & $29(56.9 \%)$ & $821(5.8 \%)$ & $<0.0001$ \\
$\begin{array}{l}\text { Other surgical procedures } \\
\quad \text { (posttransplant) }\end{array}$ & $31(60.8 \%)$ & $1516(19.1 \%)$ & $<0.0001$ \\
$\begin{array}{l}\text { Pulmonary embolism } \\
\quad \text { after listing }\end{array}$ & $5(9.8 \%)$ & $46(0.6 \%)$ & $<0.0001$ \\
$\begin{array}{l}\text { Transfusions since list- } \\
\quad \text { ing (Yes) }\end{array}$ & $49(34.3 \%)$ & $622(4.4 \%)$ & $<0.0001$ \\
\hline
\end{tabular}

recently published studies, recipients bridged with ECMO fared very poorly with a third dying within the first month and barely attaining double-digit 5-year survival rates (11\%). From a different perspective, however, the ECMO-bridged group was comprised of much sicker patients, more often requiring some form of life support. Furthermore, they had higher lung allocation scores, longer graft ischemia times, and were more likely to have had prior lung surgery (nontransplant), while on the waiting list. Finally, many of the ECMOsupported patients had refractory and severe pulmonary hypertension as indicated by the use of both inhaled and infused prostacyclins as well as nitric oxide (Table 9). This likely represents a risk factor for poor outcomes after lung transplantation [7]. In practical terms, most of these transplants should be considered "salvage" attempts. Indeed, the double-salvage strategy of using ECMO for primary graft failure followed by another lung transplant lead to dismal outcomes (Figure 2).

We also found that ECMO-bridged lung transplant recipients experienced a higher rate of posttransplant complications, including airway dehiscence, stroke, need for posttransplant dialysis, serious infections, pulmonary embolism, blood transfusion requirements, and the need for another surgical procedure. Although some of these complications can be attributed to the sicker nature of these patients, others may be direct complications of ECMO itself. Naturally, some 
Table 7. Recipient graft status.

\begin{tabular}{|c|c|c|c|}
\hline & $\begin{array}{l}\text { ECMO group } \\
(n=143)\end{array}$ & $\begin{array}{l}\text { No ECMO group } \\
\quad(n=14,120)\end{array}$ & $p$-value \\
\hline \multicolumn{4}{|l|}{ Patient/graft status } \\
\hline Alive & 52 (36.4\%) & 7093 (50.2\%) & 0.0055 \\
\hline Dead & $86(60.1 \%)$ & $6439(45.6 \%)$ & \\
\hline Lost & $2(1.4 \%)$ & $147(1.0 \%)$ & \\
\hline Retransplanted & $3(2.1 \%)$ & 441 (3.1\%) & \\
\hline Graft status & $n=131$ & $n=12781$ & \\
\hline Functioning & 96 (73.3\%) & $10766(84.2 \%)$ & 0.0006 \\
\hline Failed & $35(26.7 \%)$ & 2015 (15.8\%) & \\
\hline $\begin{array}{l}\text { Primary cause of death } \\
\text { (most common) }\end{array}$ & $n=86$ & $n=6409$ & 0.0197 \\
\hline Unknown & $3(3.5 \%)$ & $403(6.3 \%)$ & \\
\hline Other & $2(2.3 \%)$ & $366(5.7 \%)$ & \\
\hline Primary graft failure & $16(18.6 \%)$ & $310(4.8 \%)$ & \\
\hline $\begin{array}{l}\text { Hyperacute graft } \\
\text { failure }\end{array}$ & 1 (1.2\%) & $14(0.2 \%)$ & \\
\hline $\begin{array}{l}\text { Chronic rejection graft } \\
\text { failure }\end{array}$ & $5(5.8 \%)$ & 795 (12.4\%) & \\
\hline $\begin{array}{l}\text { Graft failure due to } \\
\text { infection }\end{array}$ & $1(1.2 \%)$ & $38(0.59 \%)$ & \\
\hline Bacterial septicemia & $10(11.6 \%)$ & $490(7.7 \%)$ & \\
\hline CMV infection & $0(0 \%)$ & $57(0.9 \%)$ & \\
\hline Bacterial pneumonia & $2(2.33 \%)$ & $413(6.4 \%)$ & \\
\hline $\begin{array}{l}\text { Fungal infection } \\
\text { (Aspergillus) }\end{array}$ & $3(3.5 \%)$ & $123(1.9 \%)$ & \\
\hline Infection (other) & $2(2.3 \%)$ & $83(1.3 \%)$ & \\
\hline Cardiac arrest & $4(4.7 \%)$ & $221(3.5 \%)$ & \\
\hline Ventricular failure & $2(2.3 \%)$ & $22(0.3 \%)$ & \\
\hline Cardiovascular (other) & $2(2.3 \%)$ & $47(0.7 \%)$ & \\
\hline Respiratory failure & $3(3.5 \%)$ & $820(12.8 \%)$ & \\
\hline ARDS & $1(1.2 \%)$ & $80(1.3 \%)$ & \\
\hline Pulmonary (other) & $1(1.2 \%)$ & 99 (1.5\%) & \\
\hline Stroke & $1(1.2 \%)$ & $76(1.2 \%)$ & \\
\hline $\begin{array}{l}\text { Metastatic disease } \\
\text { (other) }\end{array}$ & $3(3.5 \%)$ & $183(2.9 \%)$ & \\
\hline Multiple organ failure & $8(9.3 \%)$ & $338(5.3 \%)$ & \\
\hline Graft failure cause & $n=35$ & $n=1997$ & $<0.0001$ \\
\hline Primary nonfunction & $24(68.6 \%)$ & $537(26.9 \%)$ & \\
\hline Acute rejection & $1(2.9 \%)$ & $105(5.3 \%)$ & \\
\hline Chronic rejection & $8(22.9 \%)$ & 1065 (53.3\%) & \\
\hline Other & $2(5.7 \%)$ & $290(14.5 \%)$ & \\
\hline
\end{tabular}

exceptionally good results with ECMO bridging have been achieved in a handful of transplant centers [1-6].

Our study has some inherent limitations. First, the database is limited to the amount of detail that is provided for the ECMObridged recipients. It does not differentiate between veno-

Table 8. Rejection episodes.

\begin{tabular}{lccc}
\hline & $\begin{array}{c}\text { ECMO } \\
\text { group } \\
(\boldsymbol{n}=\mathbf{1 4 3 )})\end{array}$ & $\begin{array}{c}\text { No ECMO } \\
\text { group } \\
(\boldsymbol{n}=\mathbf{1 4 , 1 2 0})\end{array}$ & $\begin{array}{c}\boldsymbol{p} \text { - } \\
\text { value }\end{array}$ \\
\hline $\begin{array}{l}\text { Recipient acute rejection epis- } \\
\text { ode between transplant and } \\
\text { discharge }\end{array}$ & $15(10.5 \%)$ & $972(6.9 \%)$ & 0.091 \\
Treated for rejection & $\begin{array}{c}n=73 \\
n=10770\end{array}$ & \\
& $36(49.3 \%)$ & $3920(36.4 \%)$ & 0.0223 \\
\hline
\end{tabular}

Table 9. Life support.

\begin{tabular}{lccr}
\hline & $\begin{array}{c}\text { ECMO group } \\
(\boldsymbol{n}=\mathbf{1 0 5})\end{array}$ & $\begin{array}{c}\text { No ECMO group } \\
(\boldsymbol{n}=\mathbf{1 0 3 4 )}\end{array}$ & $\boldsymbol{p}$-value \\
\hline Intraortic balloon pump & $2(1.4 \%)$ & $4(0.03 \%)$ & $<0.0001$ \\
Prostacyclin infusion & $3(2.1 \%)$ & $76(0.54 \%)$ & 0.0124 \\
Prostacyclin inhalation & $1(0.7)$ & $12(0.08 \%)$ & 0.0154 \\
IV inotropes & $1(0.7 \%)$ & $0(0 \%)$ & $<0.0001$ \\
Inhaled nitric oxide & $17(11.9 \%)$ & $25(0.2 \%)$ & $<0.0001$ \\
Ventilator support & $73(51.1 \%)$ & $600(4.3 \%)$ & $<0.0001$ \\
Other life support & $8(5.6 \%)$ & $317(2.3 \%)$ & 0.0076 \\
\hline
\end{tabular}

venous and veno-arterial ECMO. Nor does it specify the length of time that patients were on ECMO support. The database also has missing data; therefore, our analysis was limited to the variables with enough data. Despite these limitations, the survival outcomes are clear. Second, there are certain important questions that this study was not able to address, including the optimal time to institute ECMO support and when bridged patients should be deactivated while on the waiting list. Third, although the ECMO-bridged recipient cohort in the present study is the largest published to date, it is a relatively small (i.e. 143 patients), compared to the nonbridged group. Despite recent publications [3-4] proposing that ECMO is safer owing to improved technologies, it is likely that better selection of patients for ECMO has also contributed to better outcomes.

Our analysis confirms that salvage ECMO for primary graft dysfunction/acute rejection followed by another lung transplant is associated with extremely poor outcomes. This finding has important implications, given the scarcity of donor lungs, we suggest that firm criteria for instituting salvage ECMO should be instituted.

Although outcomes associated with ECMO in adult patients have improved over time, its use as a bridging strategy in the sickest of lung transplant recipients has yielded relatively poor survival rates and should be carefully scrutinized with prospective trials.

\section{CONCLUSION}

The use of ECMO support as a bridge to lung transplantation is associated with significantly worse survival and more frequent postoperative complications. Therefore, we advocate very careful patient selection and cautious use of ECMO in this capacity.

\section{REFERENCES}

[1] Bittner HB, Lehmann S, Rastan A, Garbade J, Binner C, Mohr FW, Barten MJ. Outcome of extracorporeal membrane oxygenation as a bridge to lung transplantation and graft recovery. Ann Thorac Surg. 2012;94(3):942-9. doi:10.1016/j.athoracsur.2012.05.006

[2] Bermudez CA, Rocha RV, Zaldonis D, Bhama JK, Crespo MM, Shigemura N, Pilewski JM, Sappington PL, Boujoukos AJ, Toyoda Y. Extracorporeal membrane oxygenation as a bridge to lung transplant: midterm outcomes. Ann Thorac Surg. 2011;92 (4):1226-31. doi:10.1016/j.athoracsur.2011.04.122 
[3] Hoopes CW, Kukreja J, Golden J, Davenport DL, Diaz-Guzman E, Zwischenberger JB. Extracorporeal membrane oxygenation as a bridge to pulmonary transplantation. J Thorac Cardiovasc Surg. 2013;145(3):862-8. doi:10.1016/j.jtcvs.2012.12.022

[4] Fuehner T, Kuehn C, Hadem J, Wiesner O, Gottlieb J, Tudorache I, Olsson KM, Greer M, Sommer W, Welte T, Haverich A, Hoeper MM, Warnecke G. Extracorporeal membrane oxygenation in awake patients as bridge to lung transplantation. Am J Respir Crit Care Med. 2012;185(7):763-8. doi:10.1164/rccm.20110915990C

[5] Toyoda Y, Bhama JK, Shigemura N, Zaldonis D, Pilewski J, Crespo M, Bermudez C. Efficacy of extracorporeal membrane oxygenation as a bridge to lung transplantation. J Thorac Cardiovasc Surg. 2013;145(4):1065-70. doi:10.1016/j.jtcvs.2012. 12.067

[6] Lang G, Taghavi S, Aigner C, Rényi-Vámos F, Jaksch P, Augustin V, Nagayama K, Ghanim B, Klepetko W. Primary lung transplantation after bridge with extracorporeal membrane oxygenation: a plea for a shift in our paradigms for indications. Transplantation. 2012;93(7):729-36. doi:10.1097/TP.0b013e318246f8e1

[7] George TJ, Beaty CA, Kilic A, Shah PD, Merlo CA, Shah AS. Outcomes and temporal trends among high-risk patients after lung transplantation in the United States. J Heart Lung Transplant. 2012;31(11):1182-91. doi:10.1016/j.healun.2012. 07.001

\section{COMPETING INTERESTS}

We have nothing to disclose regarding this study.

\section{PUBLISHING NOTES}

(C) 2014 Shumaster et al. This work has been published open access under Creative Commons Attribution License CC BY 4.0, which permits unrestricted use, distribution, and reproduction in any medium, provided the original work is properly cited. Conditions, terms of use and publishing policy can be found at www.scienceopen.com.

Please note that this article may not have been peer reviewed yet and is under continuous post-publication peer review. For the current reviewing status please click here or scan the QR code on the right.

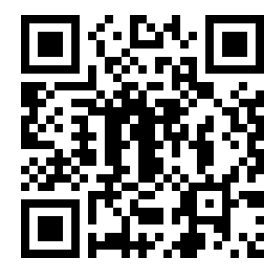

\section{ScienceOPEN.com \\ research+publishing network}

